\title{
Individual Deep Fake Recognition Skills are Affected by Viewer's Political Orientation, Agreement with Content and Device Used
}

\author{
Stefan Sütterlin ${ }^{1,2,3[0000-0002-4337-1296]}$, Torvald F. Ask ${ }^{3,4[0000-0002-1907-0004]}$, Sophia Mä- \\ gerle $^{1}$, Sandra Glöckler ${ }^{1}$, Leandra Wolf ${ }^{1}$, Julian Schray ${ }^{1}$, Alava Chandi ${ }^{1}$, Teodora \\ Bursac $^{1}$, Ali Khodabakhsh ${ }^{4[0000-0002-2873-4140]}$, Benjamin J. Knox ${ }^{4,5[0000-0002-4540-9534]}$, Mat- \\ thew Canham ${ }^{6}$, and Ricardo G. Lugo 3,4[0000-0003-2012-5700] \\ ${ }^{1}$ Faculty of Computer Science, Albstadt-Sigmaringen University, Sigmaringen, Germany \\ stefan.suetterlin@hs-albsig.de \\ ${ }^{2}$ Centre for Digital Forensics and Cyber Security, Tallin University of Technology, Tallinn, \\ Estonia \\ ${ }^{3}$ Faculty for Health, Welfare and Organization, Østfold University College, Halden, Norway \\ ${ }^{4}$ Department of Information Security and Communication Technology, Norwegian University \\ of Science and technology, Gjøvik, Norway \\ ${ }^{5}$ Norwegian Armed Forces Cyber Defense, Lillehammer, Norway \\ ${ }^{6}$ Beyond Layer Seven, LLC, United States
}

\begin{abstract}
AI-generated "deep fakes" is increasingly used by cybercriminals conducting targeted and tailored social engineering attacks, and for influencing public opinion. To raise awareness and efficiently train individuals in recognizing deep fakes, understanding individual differences in the ability to recognize them is central. Previous research suggested a close relationship between political attitudes and top-down perceptual and cognitive processing styles. In this study, we investigate the impact of political attitudes and agreement with the political message content on individual deep fake recognition skills. 163 adults (72 females $=44.2 \%)$ judged a series of video clips with politicians' statements across the political spectrum regarding their authenticity and their agreement with the message content. Half of the presented videos were fabricated via lipsync technology. In addition to agreement with each statement made, global political attitudes towards social and economic topics were assessed via the Social and Economic Conservatism Scale (SECS). There were robust negative associations between participants' general and social conservatism and their ability to recognize fabricated videos, especially when where there was agreement with the message content. Deep fakes watched on mobile phones and tablets were considerably less likely to be recognized compared to when watched on stationary computers. This is the first study to investigate and establish the association between political attitudes and interindividual differences in deep fake recognition. The study supports recently published research suggesting relationships between conservatism and perceived credibility of conspiracy theories and fake news in general. Implications for further research are discussed.
\end{abstract}

Keywords: Deep fake recognition, political orientation, social engineering. 


\section{Introduction}

In the age of increasing cyber security threats, the technological arm's race between threat actors and cybersecurity specialists spiral into material battles, where ever more sophisticated zero-day exploits are required to overcome constantly developing network defence, and vice versa. The risks attached to purchases of attractive zero-day or half-day exploits on the gray or black market, the necessity of trust, and the need for trusted double-blind auction opportunities are in principle not very different from their physical equivalents. Particularly for Advanced Persistent Threats (APT) to successfully target high- profile actors, the technological efforts that are required, the necessary expertise and/or funding required to circumvent or compromise cyber security infrastructure are not affordable for or available to many of those intending to launch a cyber attack (Meakins, 2019). As a result, the exploitation of the human factor becomes more relevant (Wang, Sun \& Zhu, 2020). The term social engineering has been described as "any act that influences a person to take an action that may or may not be in their best interest" ([www.social-engineer.com]; Hadnagy, 2018; Mouton et al., 2014). Social engineering relies on stable human traits such as for example trust, agreeableness, and conscientiousness (Uebelacker \& Quiel, 2014). The methods used to take advantage of these evolutionary rooted weaknesses (exploits) are well known tactics of persuasion (Cialdini, 1993). Persuasion tactics are particularly successful where they meet unprepared, unaware, and inexperienced individuals who do not consider themselves relevant targets, do not recognize the attack situation, and do not apply any defence strategies. Social engineering-enabled cyberattacks are estimated to account for up to $98 \%$ of all cyberattacks in 2020 (Purplesec, 2021), with absolute numbers continuously rising (IBM Security, 2020; Verizon, 2021). While social engineering attacks remain rather unchanged in regard to the human characteristics and weaknesses they target, and the persuasion techniques or cognitive vulnerabilities they exploit, their modus operandi develops parallel to technological developments and societal trends. In more recent years, social engineering attacks benefited increasingly from technological advancement. Automatized or semi-automatized opensource personality assessment allows for resource-efficient individual profiling of susceptibilities (Azucar et al., 2018; Golbeck et al., 2011; Kosinski et al., 2013), providing the ground for tailored mass-spear phishing campaigns.

Deep Fakes have the potential to become a disruptive technology changing the way we think about security aspects of virtual human-human interaction. The associated cost of DF scams was estimated to exceed 250 million USD in 2020 (iProov, 2020). The Global Trends Unit of the European Parliament associated the rise of DF with increased risks of being impersonated as an individual resulting in increased online abuse, and on a societal level contributing to political disinformation and fostering social unrest (European Parliament, 2018). AI-generated DF are becoming increasingly sophisticated and are in some cases hardly distinguishable for human eyes from 
authentic products (Korshunov \& Marcel, 2020; Rossler et al., 2019). While the market for software tools producing DF of acceptable quality keeps developing (Lyu, 2020), it provides individuals and cybercriminal gangs, as well as state actors with a constant supply to improve their arsenals. Human targets exposed to DF attacks are rendered relatively unaware, with most common cybersecurity awareness campaigns not yet preparing their customers for this attack vector. By applying DF, classic social engineering attack vectors exploiting human trust tendencies and other vulnerabilities are empowered by new technological means. The logic of social engineering, however, remains unchanged: the circumvention of technological safeguards by application of psychological means. Once a sufficient credibility of DF generation has been achieved, only little IT knowledge will be required to implement it for a specific purpose, such as impersonating a superior in a spear-phishing context. In this asymmetric context, forensic detection tools, cyber security experts with knowledge about DF risks, and comparable means of detection or mitigation are not in place due to where and how the attack can be camouflaged as unsuspicious conversation.

To date, many adversaries such as individual cybercriminals may not yet have the necessary resources, competencies or the required raw material featuring the target to produce perfect impersonifications. Available low-tech fakes bear, depending on their sophistication, familiarity with the impersonated individual, the situational context and the target person's personal vulnerabilities, an inter-individually varying risk of being detected as inauthentic. While there is a constantly progressing field of authentication tools and strategies available (e.g. Hu et al., 2021; Rossler et al., 2019), cybersecurity awareness and resilience training needs to take the individual risk factors into account in order to develop adaptive training scenarios to improve the vigilance, judgment and evaluation performance when encountering synthetic media. This training should be developed, implemented, and validated with the same care as is currently the case in phishing simulation campaigns. One precondition for efficient and effective training or awareness interventions is an understanding of the underlying human factors contributing to successful or unsuccessful differentiation between imperfect faked and authentic information. Both short-term cognitive factors such as stress, workload, and vigilance, and long-term cognitive factors related to personality and individual differences (e.g., gender, age, political orientation), expertise, and culture may affect situation awareness during a social engineering attack. This occurs by influencing the interaction between perception, working memory, decision-making, and action (Montañez et al., 2020). While recognition and judgment of faked audiovisual material without technological assistance is the major step every countermeasure against social engineering attack needs to take, there is very little knowledge about the individual differences that affect deep fake recognition. Previous social engineering attack frameworks (e.g., Uebelacker \& Quiel, 2014) have for the most part not considered the differing cognitive requirements and cognitive-emotional pro- 
cesses involved in human DF recognition, and it is only recently that these influences have started to be addressed (Montañez et al., 2020).

DF can be classified into five types: (1) face-swap/identity-swap, (2) facereenactment/puppet-mastery, (3) lip-syncing, (4) facial attribute manipulation, and (5) entire face synthesis (Masood et al., 2021) which can be separated into two main categories of facial manipulation methods (Zollhöfer et al., 2018): 1) facial expression manipulation and 2) facial identity manipulation. Glitches and minor errors in imperfect deep fakes such as asynchronous lip movements can reveal the inauthenticity. Eye-tracking studies revealed the interplay between attentional focus and imperfections of DF, resulting in an eye- tracking database provided by Gupta and colleagues (2020). The cognitive processes involved make DF recognition a perceptual task benefitting from experience and knowledge (top-down), but also determined by attentional control and shifts (bottom-up). It is therefore to be expected that marked and robust inter-individual differences in DF recognition performance exist.

To raise awareness and train individuals in recognizing the most widespread DF, the understanding of what may cause individual differences in the ability to recognize them is key for the development of educational content and methods suitable to enhance robust DF related cybersecurity awareness. While there is currently a lack of research on individual predictors of DF recognition performance, considerable knowledge exists regarding the relationships between visual perception and visual judgment, cognitive styles, and political orientation. Research on visual perception suggests powerful top-down influences on very early visual processing (Figure 1). Findings on ambiguous pictures and binocular rivalry suggest that even very early attentional processes, such as preconscious attentional resource allocation, is affected by higher-level cognitive evaluations of associated sets of stimuli. This suggests both bottom-up and knowledge-driven top-down processes from early attentional resource allocation to interpretation and evaluation of perceived patterns (e.g., Balcetis et al., 2012; Tong et al., 1998; Van Koningsbruggen et al., 2011). Even very openly laid out physical properties of the immediate physical environment one interacts with, such as distances or slopes, have been mentioned to be perceived differently depending on how much a person tries to avoid certain cognitions (Balcetis \& Dunning, 2007). Regarding the later processes of higher cognitive judgments of perceived messages and the corresponding judgment regarding their authenticity, recent research suggests a causal relationship between trait-like conservatism and belief in untrue political statements (Garrett \& Bond, 2021).

Our study aims to combine and apply the well-established work on early cognitive influences on visual perception, with more recent work on higher cognitive functioning and authenticity judgment, in the context of moderately difficult authentic or inauthentic video clips with political content. We investigated technically unaided human fake detection performance featuring politicians making political statements, where a 
part of the presented videos has been faked via lip synchronization (facial expression manipulation).

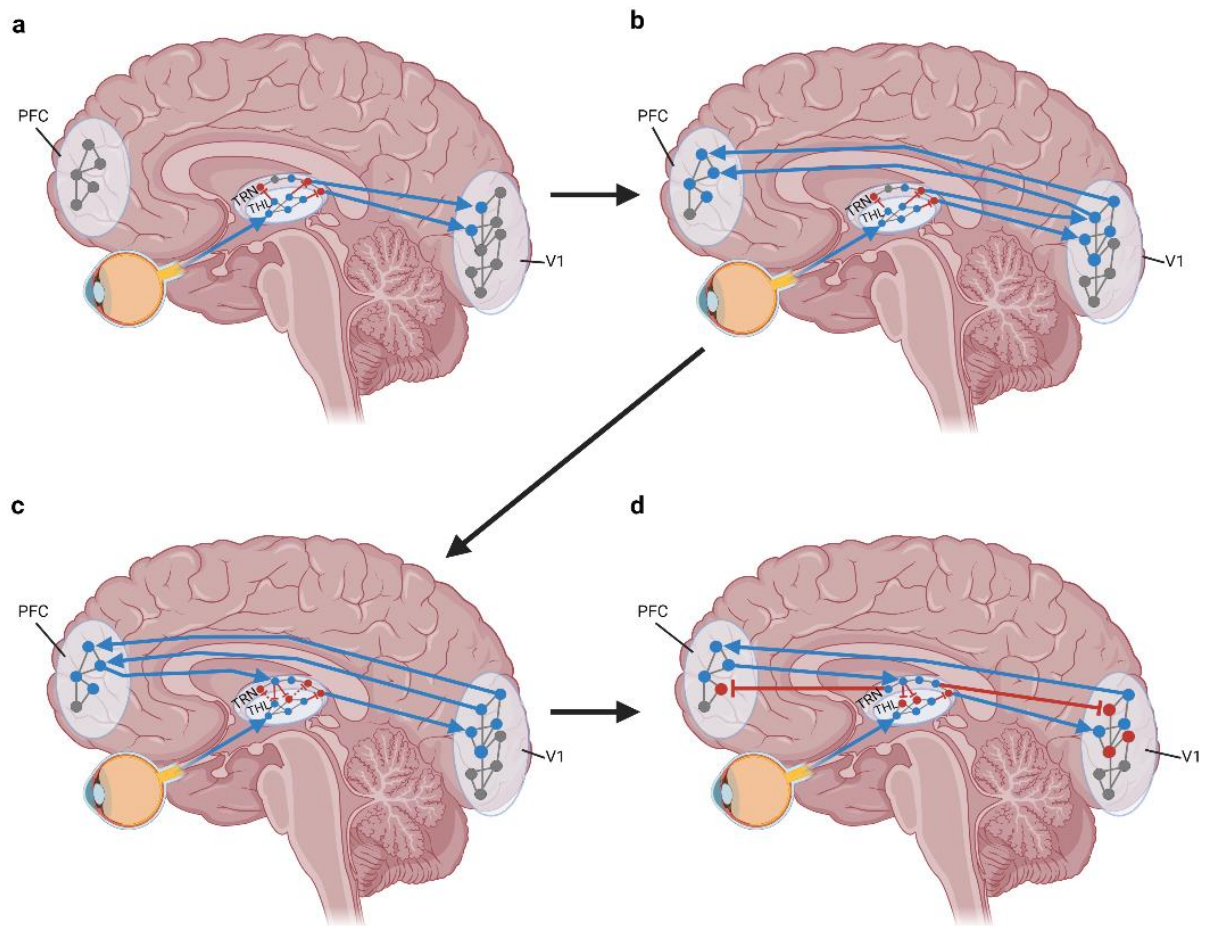

Fig. 1. Visual representation of top-down perceptual filtering. The PFC exerts top-down control on perception by changing the activity in TRN which serves as an inhibitory filter between preconscious and conscious perceptual processing (Nakajima et al., 2019; Philips et al., 2016; Pinault, 2004). a Visual stimuli enter the eye and are processed in the THL before being relayed through the TRN filter to enter conscious processing in V1. b Perceptual information is sent from visual cortices to the PFC where it is represented in working memory and processed according to perceptual goals. $\mathbf{c}$ The PFC combines perceptual information from V1 with taskrelated information to change how the TRN filters visual information from the THL to V1. $\mathrm{d}$ Input from V1 to PFC is altered according to top-down control. $\mathrm{PFC}=$ prefrontal cortex. THL $=$ Thalamus. TRN $=$ Thalamic reticular nucleus. V1 $=$ Primary visual cortex. Blue dots $=$ activated nodes. Blue arrows $=$ Excitatory projections. Red dots $=$ inhibited nodes. Red lines $=$ inhibitory projections.

To the best of our knowledge, this is the first study to investigate the relationship of visual perception in DF contexts and political orientation. We took into account that respondents may use smartphones, tablets or desktop/laptop devices when responding 
to the online survey. Remote social engineering techniques can be expected to be more successful, where situational awareness is reduced due to for example smaller screen size, or simultaneous parallel activities (multitasking) (Canham et al., 2022). In order to determine and quantify if and how much device choices impact the individual and momentary cybersecurity risk profile and may thus inform attackers choice of attack vectors and implementation of social engineering attacks, device choice effects need to be taken into account. We thus investigated the device choice effect on the relationship between political attitude and specific statement agreement on DF recognition performance.

In sum, we hypothesized (Hypothesis $\mathrm{H}_{1}$ ) trait conservatism to be negatively associated with successful identification of videos as authentic or faked; we also expected to see that the subjectively perceived probability of authenticity was positively associated with trait conservatism (Hypothesis $\mathrm{H}_{2 \mathrm{a}}$ ) and the agreement with the video's spoken message content (Hypothesis $\mathrm{H}_{2 b}$ ). Finally, we expected overall recognition performance to be lower on smartphones and tablets compared to laptops or desktop devices (Hypothesis $\mathrm{H}_{3}$ ).

\section{Methods}

\subsection{Ethics}

The present study complies with the Declaration of Helsinki and is in line with the Recommendations for the Conduct, Reporting, Editing and Publication of Scholarly Work in Medical Journals. Participants gave their informed consent prior to the study and were debriefed about the study's purpose after completing the data collection. No deception was taking place. Participants were informed that they could withdraw from participation at any time. All participation was anonymous, no IP address or any personal information that could lead to identification of participants was registered at any point. It was made clear to all participants that some of the videos would be faked and do not represent the view of the impersonated public figure.

\subsection{Participants and recruitment}

Literature research on related topics of knowledge-driven visual perception resulted in a conservatively expected estimated effect size of partial $\mathrm{f} 2=.15$. Following common conventions, the probability of a type-I-error was set at $\alpha=0.05$ and for type-II-errors $\beta=0.20$. Based on the choice of appropriate analytical tools these settings resulted in a recommended sample size of 68 participants ( $G^{*}$ Power; Faul et al., 2009). A convenience sample of 164 participants was recruited via social media, where 163 ( $n_{\text {female }}$ $=72 ; 44.2 \%$ ) participants completed the survey and were included in the further analysis. Data was collected via Google Forms. 


\subsection{Design and procedure}

A correlational approach based on data obtained in an online-survey from individuals recruited via social media, students and staff from a university of applied sciences was followed. The university has a portfolio of courses within the areas of economy, technology and life sciences. Participants provided basic socio-demographic data and were presented with twelve video clips of 10 to 26 seconds duration. The clips were presented in randomized order and contained brief political statements by German politicians covering the breadth of the political spectrum following a consensus decision of the authors and categorization into left-wing, neutral and right-wing political orientations. Five videos were faked, seven were authentic. Participants were asked about the authenticity of the videos (10-point Likert-scale ranging from (" 1 = certainly not authentic" to "10 = certainly authentic") and about the degree to which they agreed with the political statement that was made (4-point Likert-scale ranging from "agree not at all" to "fully agree"). General conservative traits were also assessed. Following the survey, participants were debriefed in more detail about the purpose of the study and had the opportunity to leave an email address to participate in a lottery for five prizes in the value of 20 EUR.

\subsection{Equipment and measures}

The 12-Item Social and Economic Conservatism Scale (SECS; Everett, 2013) was used for measuring conservatism. The scale consists of 12 items that are rated on a 0 100 scale by asking "How positive or negative do you feel about each issue on the scale". The total score as well as two subscales, economic and social conservatism, can be computed. Sample items for the social conservatism subscale ( 7 items) include 'abortion, limited government, traditional marriage' and for the economic conservatism subscale (5 items) include 'tax, welfare benefits, fiscal responsibility'. The scale shows good overall reliability (Cronbach's $\alpha=.88$ ). For this study, the SECS was translated to German and showed acceptable reliability (Cronbach's $\alpha=.71$ ).

\section{$2.5 \quad$ Videos}

Lip-sync approaches take an arbitrary audio track as input and use it to derive a video of a target person with matching mouth region movements. The audio track can be an authentic recording, an audio- deepfake recording with a transformed identity (a.k.a. voice conversion) or be generated by text-to-speech synthesis. Deep Fakes were made using Wav2Lip by uploading one audio track (in .wav format) and one video track (in .mp4 format) per DF. Wav2Lip analyses the mouth area and adapts the mouth movement to the new fake sound, the old sound is replaced by the new one. First, videos of real politicians who talk slowly, move little, and do not open their mouths wide were 
collected. Then audio with similar voices or the voice of the same person with a different statement were collected. The correct percentage of identified fake videos and participant agreement with the content of the video were used as dependent variables. Deep Fake Identification (DFI) was computed as a binary variable and correct identification percentages were computed. Participant agreement with the video content was measured on a X-point Likert scale (do not agree- totally agree) and averages for both real and fake videos were computed. Reliability was acceptable for both the real videos (Cronbach's $\alpha=.79$ ) and the fake videos (Cronbach's $\alpha=.68$ ).

\subsection{Pilot study}

A pilot study with 19 video clips was conducted and a community sample of 39 persons were recruited. Detection performance, means and distributions were analyzed in order to select five clips that showed medium difficulty and covered the whole range of the political spectrum (two left-wing, two right-wing, one neutral). This selection was included into the main study (see below).

\subsection{Data reduction and analysis}

Statistical analysis was done with JASP version 14.1 (Gross-Sampson, 2020). All variables were checked for normality. Where criterion was not met, non-parametric alternatives were chosen. Bivariate correlations were calculated, and all variables were entered in the calculation. For the regression analysis, the conservatism scale including its subscales (SECS) was entered as the independent variable and Deepfakes as the dependent variable. To test the influence of pc/mobile device on DF identification, non-parametric independent samples test was used (Mann-Whitney $\mathrm{U}$ ).

\section{Results}

Descriptive statistics and correlations between conservatism, detection accuracy, and agreement with content can be found in table 1 .

\section{1 $\quad H_{1}$ : Conservativism and deep fake identification}

To test if being more conservative (SECS scores) predicted less correct video clip categorization (i.e., recognizing the status as being an authentic or faked/synthetic video), a regression analysis was performed where the SECS sub scales were entered as the independent variables and the number of correct deep fake identifications was entered as the dependent variable.

The SECS score was associated with more correct identifications $\left(R^{2}=.056, F=\right.$ $7.22, p=.01$; Figure 2 ). The association between conservatism and correct identifications was due to a significant correlation between the SECS social subscale (beta = - 
.267, $t=-3.08, p=.002)$, while SECS subscale on economic conservatism remained insignificant (beta $=.129, t=1.49, p=.139$ ).

Table 1. Descriptive statistics and correlations between conservativism, detection accuracy, and agreement with content $(N=163)$.

\begin{tabular}{|c|c|c|c|c|c|c|c|c|c|c|c|}
\hline & Mean & SD & Min & Max & 1 & 2 & 3 & 4 & 5 & 6 & 7 \\
\hline 1. SECS total & 5.10 & 1.08 & 2.92 & 8.00 & & & & & & & \\
\hline 2. Social subscale & 5.17 & 1.45 & 1.43 & 8.57 & $.947^{* * * *}$ & & & & & & \\
\hline 3. Economic subscale & 5.01 & 0.94 & 2.80 & 8.40 & $.685^{* * *}$ & $.449^{* * *}$ & & & & & \\
\hline 4. Correct identification & 7.73 & 2.29 & 0.00 & 12.00 & $-.203^{* *}$ & $-.241^{* *}$ & -.010 & & & & \\
\hline $\begin{array}{l}\text { 5. Authenticity rating of } \\
\text { authentic videos }\end{array}$ & 3.49 & 0.45 & 2.00 & 4.00 & -.053 & -.136 & $.175^{*}$ & $.609^{* * *}$ & & & \\
\hline $\begin{array}{l}\text { 6. Agreement with con- } \\
\text { tent in real videos }\end{array}$ & 1.75 & 0.31 & 1.00 & 3.00 & $-.299^{* * * *}$ & $-.305^{* * * *}$ & $-.185^{*}$ & $.286^{* * *}$ & $.264^{* * * *}$ & & \\
\hline $\begin{array}{l}\text { 7. Authenticity rating of } \\
\text { faked videos }\end{array}$ & 2.42 & 0.90 & 1.00 & 4.00 & $.193^{* *}$ & .147 & $.184^{*}$ & $-.576^{* * *}$ & .000 & -.111 & \\
\hline $\begin{array}{l}\text { 8. Agreement with con- } \\
\text { tent in fake videos }\end{array}$ & 1.66 & 0.31 & 1.00 & 3.00 & .123 & .100 & -.148 & -.150 & .134 & .128 & $.355^{* * *}$ \\
\hline
\end{tabular}

Notes. Non-parametric correlations (Spearman's rho). SECS = Social and Economic Conservativism Scale. $* p<.050 . * * p<.010 . * * * p<.001$.

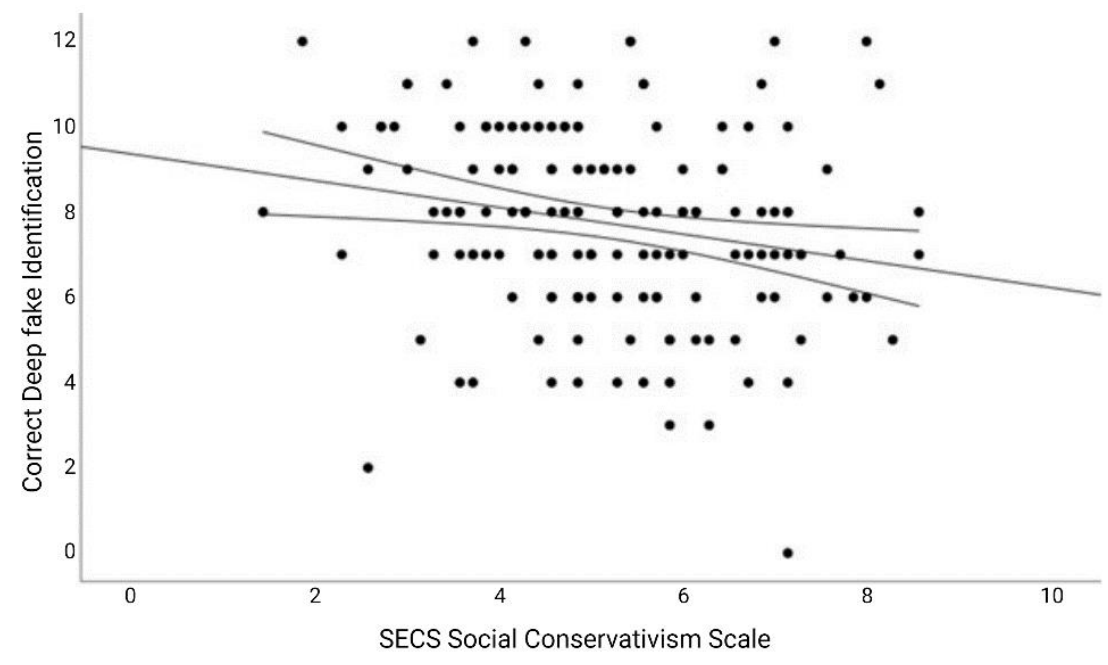

Fig. 2. Scatterplot with linear regression line showing correlation between social conservativism and number of correctly classified video clips. Confidence intervals to the mean. 
To test if being more conservative (SECS) was associated with the authenticity ratings and agreement of spoken content, regression analyses were performed where the SECS sub scales were entered as the independent variables and video ratings and agreement with the statements made in the videos for both the real $\left(\mathrm{H}_{2 \mathrm{a}}\right)$ and fake $\left(\mathrm{H}_{2 \mathrm{~b}}\right)$ videos were entered as the dependent variables.

In line with previous research, age showed positive trends of being associated with conservatism ( rho $=.141, p=.073$ ). When controlled for age (beta $=-.063, t=-.808$, $p=.420)$, the effect of conservatism on deep fake recognition persisted $\left(R^{2}=.060, F\right.$ $=3.37, p=.020$ ), suggesting that age-typical media consumption habits or ageaffected IT-literacy did not explain the effect.

\section{2 $\mathrm{H}_{2 \mathrm{a}}$ : Effect of conservativism on judgment of videos' degree of authenticity}

For authentic videos, trait conservatism measured as SECS total score predicted perceived authenticity $\left(R^{2}=.067, F=5.75, p=.004\right)$, where both the social (beta = $.214, p=.014$ ) and the economic subscales (beta $=.275, p=.002$ ) were significant predictors.

For faked videos, trait conservatism predicted higher authenticity ratings $\left(R^{2}=\right.$ $.042, F=3.49, p=.033$ ). While both scales were not significant predictors when analysed separately (SECS social: beta $=.076, t=.868, p=.387$ ), the economic conservatism (beta $=.158, t=1.81, p=.072$ ) did show some weak tendencies of being associated with higher authenticity ratings of faked videos for fake video ratings.

\section{3 $\mathrm{H}_{2 \mathrm{~b}}$ : Effect of agreement with message content on judgment of video's degree of authenticity}

Participants who agreed more with the message content of authentic videos, judged them to be more authentic $(r=.264, p<.001$; Table 1$)$. Participants who agreed more with the message content of faked videos, rated them also as more likely to be authentic $(r=.355, p<.001$; Table 1$)$.

\subsection{H3: Influence of device used on authenticity judgment accuracy}

Figure 3 shows the result from the Mann-Whitney $U$ tests. People identified more deep fakes correctly $(U=1821.50, Z=2.42, p=.016)$ when using a laptop or desktop device $\left(N=110 ; M_{\text {rank }}=82.94\right)$ over a mobile phone or tablet $\left(N=44 ; M_{\text {rank }}=63.90\right)$. Using a laptop or desktop $\left(M_{\text {rank }}=81.96\right)$ led to higher levels of perceived authenticity ratings $(U=1929.00, Z=1.98, p=.047)$ than using phones or tablets $\left(M_{\mathrm{rank}}=66.34\right)$. More female participants used mobile phones or tablets $(66 \%)$ compared to males $\left(\chi^{2}\right.$ $=6.09, d f=1, p=.019)$. 


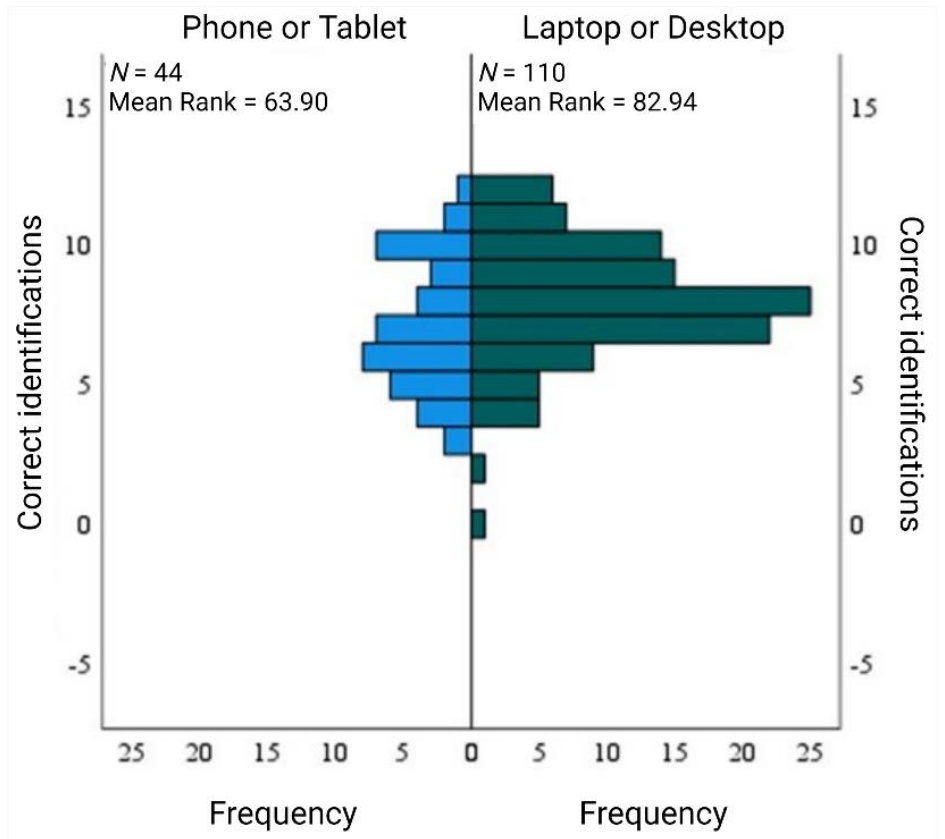

Fig. 3. Comparisons of deep fake recognition accuracy between phones/tablets and laptop/desktop devices.

\section{Discussion}

This research investigated how general political orientation on the conservatism domain is associated with detection accuracy of deep faked videos. Due to the novelty of the topic of individual differences in DF recognition, we consider this a first pilot study into a new thematic topic area which is likely to trigger more research activity in the future.

Previous research indicated a positive relationship between trait conservatism and the perceived credibility of conspiracy theories (Garret \& Bond, 2021). Our study aimed to test this hypothesis in the more specific field of deep fake recognition, which is currently an increasingly relevant element of political dis-/misinformation as well as the spread of conspiracy theories. Our results suggest a moderate, but statistically significant relationship between conservatism and deep fake recognition. According to these initial results, more socially (but not economically) conservative political views were associated with more susceptibility to believing that video clips to be authentic. This association between trait conservatism and deep fake recognition performance seemed to be carried by the individual's extent of agreement with the politi- 
cal statement that is transported in the video. There was a positive linear relationship between agreeing with the spoken message content and the degree to which the message transporting video was considered authentic. While this association was found for both authentic and faked videos, the association was slightly (but not significantly) stronger for the category of faked videos.

This research also showed that using a mobile phone increased DF susceptibility. Using a mobile phone led to higher failure rates in detecting DF, thus supporting the hypothesis. This may in part be explained by the fact that humans tend to perform worse when detecting DF in videos with smaller screen size or due to artifacts from the faked videos with low resolution being masked by the quality compression (Rossler et al., 2019). Research on susceptibility to remote online social engineering (ROSE) attacks suggest a higher vulnerability when portable devices, in particular smartphones, are used (Powell \& Canham, 2021). This was confirmed to be also true for DF recognition in this study. It was beyond the scope of this study to investigate causal pathways and disentangle them, but we consider it plausible to assume that both factors, the reduced situational awareness resulting from (1) smaller screens and (2) multitasking/distraction may have contributed. Since smaller screens are partially compensated by the closer distance to the eye and thus a similar visual field angle, perhaps (2) is more likely to be true and should be considered in future studies to inform human-centered security-relevant design.

While gender effects were not part of the initial research question, preliminary analyses indicate that female participants reported significantly more mobile phone use than males $(66 \%)$. Taken with the findings from the first hypothesis that conservatism would be associated with DF identification, this could explain why females performed worse than males on DF detection, but differences are not significant or have small effect sizes and are thus not included here in detail. These findings add to the previous research that gender might not influence DF detection (Montañez et al., 2020). Gender effects have not been reported before in DF recognition. Previous research suggested women might be more susceptible to social engineering (e.g., Halevi et al., 2015; Lin et al., 2019; Sheng et al., 2010) but previous studies mainly focused on personality traits and susceptibility to phishing emails.

The effects from this study are in line with very recent findings on the inaccuracy in regards to the judgment of political statements' truth and conservatism in a USAmerican sample (Garrett \& Bond, 2021). It also gives support to recent literature on top-down processes of visual perception (Aitken et al., 2020a, 2020b; Panichello \& Turk-Browne, 2021). The participants were clearly instructed to judge the authenticity and can be assumed to have searched for technical glitches, inconsistencies, spectral artifacts, and aberrations. These perceptions have to be compared with internal cognitive representations of what a hypothetical "normal" (authentic) standard model could be. 


\subsection{Limitations}

This study has several limitations that could have influenced the results. The participants were recruited through convenience sampling and from a specific region in Germany and are thus not necessarily representative on a national or international level. Also, affinity for information technology (IT) was not assessed in the sample. People more accustomed to using or being exposed to IT media might have a better understanding of social media influence and social engineering. However, previous research on other deceptive means applied in cybercrimes, such as phishing simulations, suggest that IT-affinity may be related to overconfidence and is not per se a relevant protective factor (Pattinson et al., 2012). The variable of age, which is commonly related to IT-affinity, did not have any statistically significant impact on the overall findings.

While there were significant findings both at group levels and at the gender levels, effect sizes were small. The sample size met the power analysis requirements, but the small yet significant findings could be due to the relationship of the variables is not as strong as hypothesized, the fact that larger sample sizes produce smaller (and more accurate) effects, or that the sample was homogenous in their location, resulting in a restriction-of-range effect.

\subsection{Future research}

The present findings have to be replicated in other countries, with larger samples, and preferably with an equal distribution of pads, laptops, and mobile phones between genders in order to make conclusions about gender effects. The nature of this survey and the informed consent required for data collection and processing prepares the participants for the challenge and induces a critical evaluation from their side. In real life conditions, targets of DF-related social engineering attacks are unlikely to be prepared and may have a certain level of personal knowledge and trust in relation to the individual shown. Thus, the findings of this study may have stronger relevance for the area of political disinformation than individual social engineering as it is carried out in for example spear-phishing attacks.

To investigate perceptual processes underlying individual differences in DF recognition closer, eye- tracking may be a useful tool to assess whether areas in the videos that contain synthetic artifacts are visually scanned equally between individuals that perform well, compared to individuals that do not perform well on the DF detection task. It may also be necessary to pair eye-tracking data with EEG recordings to see if there is a difference in DF artifact-detection associated responses (event related potentials) between participants who are visually scanning the same areas of the DF. This will be important to determine if top-down knowledge (e.g. expectations influenced by conservativism) affects perceptual processes at the attention allocation- or encoding-level of DF stimuli processing. Dissociating the effects of attention and expecta- 
tion on early sensory processing is not straightforward (Rungratsameetaweemana \& Serences, 2019) and should be properly addressed in future research. Comparing the differences in DF detection between DF facial identity manipulation techniques and facial expression manipulation techniques may go further in demonstrating the extent to which top- down processes influence perceptions. Facial identity manipulation DF may have less of the expressive characteristics of the impersonated individual, or a timing of expressive behaviors that are a little off from the impersonated individual, thus providing additional clues that may give away the DF. To pick up on these characteristics will, however, require that participants are somewhat familiar with the impersonated individual and pose new methodological challenges for future research designs. Lastly, very recent research suggests that the relationship between conservatism and belief in conspiracy theories is partially explained by the personality trait of conscientiousness (Lawson \& Kakkar, 2021). Despite the conceptual differences between the concept of beliefs into conspiracy theories on one hand, and the very perceptual task of recognizing technical artifacts in deep fakes, future studies should consider these findings and the effects personality traits such as conscientiousness have on cognitive processing.

It was beyond the scope of this study to investigate cognitive-perceptual pathways on an intraindividual level, as this would have required techniques such as gazetracking and time-sensitive stimulus presentation. Future research might thus target more on these covert processes and their interaction with political attitudes. Since the present study used the DF technique of facial expression manipulation (lip syncing). Not being familiar with the politician impersonated in a DF will reduce the likelihood of detecting micro expressions that are incongruent with that politician's usual facialexpressive behaviors. Conversely, not being familiar with the politicians in the videos could perhaps go further in isolating the effects of the interaction between participant's political attitudes and the political message in a DF video on perceptual processing. Nevertheless, we believe that the effects related to being familiar with the politician in the DF compared to not being familiar with the politician in the DF should be addressed sufficiently by using a mix of known and unknown politicians.

The presented results regarding political attitudes and agreement with message content may inform future research on individual perception and recognition processes. It was beyond the scope of this online survey to investigate cognitive processes during video presentation, as this would require standardized laboratory conditions and a higher degree of repetition. Nevertheless, the identified variables my play a role in the future identification of individual cognitive processing styles. Tahir et al. (2021) used gaze-tracking and self-report to understand the detection perceptual process on DF data. They discovered that humans tend to focus on the major regions of the face such as eyes, hair, and nose, and report eyes, nose, forehead, lips, cheeks, and expression as the visual cues they used for detection. In a similar study on face-swap data by Wohler et al. (2021), the participants fixated on mouth and nose and reported 
blur artifacts, contour artifacts, and unnatural expressions or eye movements. Participants in another study by Thaw et al. (2020) self-reported artifacts such as lack of expressions, lack of emotions, and unnatural behavior as well as flickering, blurred faces, rendering artifacts, and abnormal mouth movements as justification for their decision. Findings from political psychology suggest characteristic stimulus processing, priorisation, risk proneness and sensitivity to threatening stimuli in conservative persons compared to those with more liberal attitudes (Schreiber et al., 2012). Future research my proceed with combining perceptual psychology and research on political attitudes with deep fake recognition skills in order to explain political phenomena related to disinformation and conspiracy believes, and thus inform the recent efforts into the development of countermeasures (see e.g., Compton et al., 2021).

\section{Conclusion}

The study suggests a statistically significant association between conservatism and the ability to recognize deep fakes. A driving factor for this effect appears to be the level of agreement with the political message transported by the synthetic media. Portable devices such as phones have a deteriorating effect on deep fake recognition skills. The study is in line with recent research on conservatism and perceived credibility of political disinformation and conspiracy theories. While effect sizes being small to moderate, this study may inform future research to investigate relevant and novel psychological aspects in order to provide a more comprehensive overview over influencing factors that could explain social engineering processes and vulnerabilities targeted by synthetic media.

Acknowledgements. This study was supported by the Norwegian Research Council (project number 302941). A preprint of this article is available at PsyArXiv (Sütterlin et al., 2021).

\section{References}

1. Aitken, F., Menelaou, G., Warrington, O., Koolschijn, R. S., Corbin, N., et al. (2020a). Prior expectations evoke stimulus-specific activity in the deep layers of the primary visual cortex. PLOS Biology, 18(12), e3001023.

2. Aitken, F., Turner, G., \& Kok, P. (2020b). Prior expectations of motion direction modulate early sensory processing. The Journal of Neuroscience, 40(33), 6389-6397.

3. Azucar, D., Marengo, D., \& Settanni, M. (2018). Predicting the Big 5 personality traits from digital footprints on social media: A meta-analysis. Personality and Individual Differences, 124, 150-159.

4. Balcetis, E., \& Dunning, D. (2007). Cognitive dissonance and the perception of natural environments. Psychological Science, 18(10), 917-921. 
5. Balcetis, E., Dunning, D., \& Granot, Y. (2012). Subjective value determines initial dominance in binocular rivalry. Journal of Experimental Social Psychology, 48(1), 122-129.

6. Canham, M., Posey, C., \& Constantino, M. (2022). Phish Derby: Shoring the Human Shield Through Gamified Phishing Attacks. Frontiers in Higher Education. Advance Online Publication.

7. Cialdini, R. B. (1993). The psychology of persuasion. New York.

8. Compton, J., van der Linden, S., Cook, J., \& Basol, M. (2021). Inoculation theory in the post- truth era: Extant findings and new frontiers for contested science, misinformation, and conspiracy theories. Social and Personality Psychology Compass, 15(6), e12602.

9. European Parliament (2018). Global Trendometer. Essays on medium- and long-term global trends. ISSN 2529-6345.

10. Everett, J. A. (2013). The 12 item social and economic conservatism scale (SECS). Plos One, 8(12), e82131.

11. Garrett, R. K., \& Bond, R. M. (2021). Conservatives' susceptibility to political misperceptions. Science Advances, 7(23), eabf1234.

12. Golbeck, J., Robles, C., \& Turner, K. (2011). Predicting personality with social media. In CHI'11 extended abstracts on human factors in computing systems (pp. 253-262).

13. Goss-Sampson, M. A. (2020). Statistical Analysis in JASP 0.14: A Guide for Students. November 2020.

14. Gupta, P., Chugh, K., Dhall, A., \& Subramanian, R. (2020, October). The eyes know it: Fakeet-an eye-tracking database to understand deepfake perception. In Proceedings of the 2020 International Conference on Multimodal Interaction (pp. 519-527).

15. Hadnagy, C. (2018). Social engineering: The science of human hacking. John Wiley \& Sons.

16. Halevi, T., Memon, N., \& Nov, O. (2015). Spear-phishing in the wild: A real-word study of personality, phishing self-efficacy and vulnerability to spear-phishing attacks. Social Science Research Network.

17. Hu, S., Li, Y., \& Lyu, S. (2021). Exposing GAN-Generated Faces Using Inconsistent Corneal Specular Highlights. ICASSP 2021 - 2021 IEEE International Conference on Acoustics, Speech and Signal Processing (ICASSP).

18. IBM Security (2020). Cost of a data breach report 2020. Retrieved from: https://www.capita.com/sites/g/files/nginej291/files/2020-08/Ponemon-Global-Cost-ofData-Breach- Study-2020.pdf

19. iProov (2020). The Threat of Deepfakes. The consumer view of deepfakes and the role of biometric authentication in protecting against their misuse. Retrieved from: https://www.iproov.com/wp- content/uploads/2021/05/iProov-Deepfakes-Report.pdf

20. JASP Team (2020). JASP (Version 0.14.1)

21. Korshunov, P., \& Marcel, S. (2020). Deepfake detection: humans vs. machines. arXiv preprint arXiv:2009.03155.

22. Kosinski, M., Stillwell, D., \& Graepel, T. (2013). Private traits and attributes are predictable from digital records of human behavior. Proceedings of the National Academy of Sciences, 110(15), 5802-5805.

23. Lawson, M. A., \& Kakkar, H. (2021). Of pandemics, politics, and personality: The role of conscientiousness and political ideology in the sharing of fake news. Journal of Experimental Psychology: General. Advance online publication. 
24. Lin, T., Capecci, D. E., Ellis, D. M., Rocha, H. A., Dommaraju, S., Oliveira, D. S., \& Ebner, N. C. (2019). Susceptibility to Spear-Phishing Emails: Effects of Internet User Demographics and Email Content. ACM transactions on computer-human interaction : a publication of the Association for Computing Machinery, 26(5), 32. https://doi.org/10.1145/3336141

25. Lyu, S. (2020, July). Deepfake detection: Current challenges and next steps. In 2020 IEEE International Conference on Multimedia \& Expo Workshops (ICMEW) (pp. 1-6). IEEE.

26. Masood, M., Nawaz, M., Malik, K. M., Javed, A., \& Irtaza, A. (2021). Deepfakes Generation and Detection: State-of-the-art, open challenges, countermeasures, and way forward. arXiv preprint arXiv:2103.00484.

27. Meakins, J. (2019). A zero-sum game: the zero-day market in 2018. Journal of Cyber Policy, 4(1), 60-71.

28. Montañez, R., Golob, E., \& Xu, S. (2020). Human cognition through the lens of social engineering cyberattacks. Frontiers in Psychology, 11, 1755.

29. Mouton, F., Leenen, L., Malan, M. M., and Venter, H. S. (2014). Towards an ontological model defining the social engineering domain. 11th Human Choice and computers International Conference, pp. 266-279.

30. Nakajima, M., Schmitt, L. I., \& Halassa, M. M. (2019). Prefrontal Cortex Regulates Sensory Filtering through a Basal Ganglia-to-Thalamus Pathway. Neuron, 103(3), 445-458.

31. Panichello, M. F., \& Turk-Browne, N. B. (2021). Behavioral and Neural Fusion of Expectation with Sensation. Journal of Cognitive Neuroscience, 33(5), 814-825.

32. Pattinson, M., Jerram, C., Parsons, K., McCormac, A., \& Butavicius, M. (2012). Why do some people manage phishing e-mails better than others? Information Management and Computer Security, 20(1), 18-28.

33. Phillips, J. M., Kambi, N. A., \& Saalmann, Y. B. (2016). A Subcortical Pathway for Rapid, Goal-Driven, Attentional Filtering. Trends in Neurosciences, 39(2), 49-51.

34. Pinault, D. (2004). The thalamic reticular nucleus: structure, function and concept. Brain Research Reviews, 46(1), 1-31.

35. Powell, K., \& Canham, M. (2021). User Be Aware: Is your Smart Phone or TV Putting You at Risk? Proceedings of the 65th Annual Meeting of the Human Factors and Ergonomics Society, Santa Monica, CA: Human Factors and Ergonomics Society.

36. Purplesec. (2021). 2021 Cyber Security Statistics. The Ultimate List Of Stats, Data \& Trends. Retrieved from: https://purplesec.us/resources/cyber-security-statistics/

37. Rossler, A., Cozzolino, D., Verdoliva, L., Riess, C., Thies, J., \& Niessner, M. (2019). FaceForensics++: Learning to Detect Manipulated Facial Images. 2019 IEEE/CVF International Conference on Computer Vision (ICCV).

38. Rungratsameetaweemana, N., \& Serences, J. T. (2019). Dissociating the impact of attention and expectation on early sensory processing. Current opinion in psychology, 29, 181186.

39. Schreiber, D., Fonzo, G., Simmons, A. N., Dawes, C. T., Flagan, T., Fowler, J. H., \& Paulus, M. P. (2013). Red brain, blue brain: Evaluative processes differ in Democrats and Republicans. PLos One, 8(2), e52970.

40. Sheng, S., Holbrook, M., Kumaraguru, P., Cranor, L., \& Downs, J. (2010). Who Falls for Phish? A Demographic Analysis of Phishing Susceptibility and Effectiveness of Interventions. 28th ACM Conference on Human Factors in Computing Systems, 373-382. 
41. Sütterlin, S., Ask, T. F., Mägerle, S., Glöckler, S., Wolf, L., Schray, J., Chandi, A., Bursac, T., Khodabakhsh, A., Knox, B. J., Canham, M., \& Lugo, R. G. (2021). Individual deep fake recognition skills are affected by viewers' political orientation, Agreement with Content and Device Used. PsyArXiv. https://doi.org/10.31234/osf.io/hwujb

42. Tahir, R., Batool, B., Jamshed, H., Jameel, M., Anwar, M., Ahmed, F., ... \& Zaffar, M. F. (2021, May). Seeing is Believing: Exploring Perceptual Differences in DeepFake Videos. In Proceedings of the $2021 \mathrm{CHI}$ Conference on Human Factors in Computing Systems (pp. 1-16).

43. Thaw, N. N., July, T., Wai, A. N., Goh, D. H. L., \& Chua, A. Y. (2020). Is it real? A study on detecting deepfake videos. Proceedings of the Association for Information Science and Technology, 57(1), e366.

44. Tong, F., Nakayama, K., Vaughan, J. T., \& Kanwisher, N. (1998). Binocular rivalry and visual awareness in human extrastriate cortex. Neuron, 21(4), 753-759.

45. Uebelacker, S., \& Quiel, S. (2014, July). The social engineering personality framework. In 2014 Workshop on Socio-Technical Aspects in Security and Trust (pp. 24-30). IEEE.

46. Van Koningsbruggen, G. M., Stroebe, W., \& Aarts, H. (2011). Through the eyes of dieters: Biased size perception of food following tempting food primes. Journal of Experimental Social Psychology, 47(2), 293-299.

47. Verizon (2021). DBIR 2021 Data breach investigations report. Retrieved from: https://www.verizon.com/business/en-gb/resources/reports/dbir/

48. Wang, Z., Sun, L., \& Zhu, H. (2020). Defining social engineering in cybersecurity. IEEE Access, 8, 85094-85115.

49. Wöhler, L., Zembaty, M., Castillo, S., \& Magnor, M. (2021, May). Towards Understanding Perceptual Differences between Genuine and Face-Swapped Videos. In Proceedings of the 2021 CHI Conference on Human Factors in Computing Systems (pp. 1-13).

50. Zollhöfer, M., Thies, J., Garrido, P., Bradley, D., Beeler, T., Pérez, P., ... Theobalt, C. (2018). State of the Art on Monocular 3D Face Reconstruction, Tracking, and Applications. Computer Graphics Forum, 37(2), 523-550. 